2

3 Identification of polymethylene-interrupted polyunsaturated fatty acids (PMI-PUFA) by solvent-

4 mediated covalent adduct chemical ionization triple quadrupole tandem mass spectrometry

5 Dong Hao Wanga, ${ }^{a}$, Zhen Wang ${ }^{a, b}$, J. Raven Cortright ${ }^{a}$, Kim Phuong Le ${ }^{a}$, Lei Liu ${ }^{a, c}$, K. S. D. Kothapallia*,

6 J. Thomas Brenna $a^{a, b, d *}$

7

8

a. Dell Pediatric Research Institute, Depts of Pediatrics, of Chemistry, and of Nutrition, University of

9 Texas at Austin, 1400 Barbara Jordan Blvd, Austin, TX 78723, United States

b. Department of Food Science, Cornell University, Ithaca, NY 14850, United States

c. College of Veterinary Medicine, Hunan Agricultural University, Changsha, Hunan Province 410128, 
Supplementary Table 1. List of observed and expected SMCI-MS/MS diagnostic ions for PMI-FAME with $\Delta 5$ isolated double bond ( $\omega$ diagnostic ions generalized). . .3

Supplementary Table 2. List of expected SMCI-MS/MS diagnostic ions for PMI-FAME with $\Delta 4$ isolated double bond.

Supplementary Table 3. List of expected SMCI-MS/MS diagnostic ions for PMI-FAME with $\Delta 6$ isolated double bond.

Supplementary Table 4. List of observed and expected SMCI-MS/MS diagnostic ions for PMI-FAME with $\Delta 7$ isolated double bond.

Supplementary Figure 1. Identification of $14: 1(\Delta 5)$ in a ruminant milk sample by CACl-MS/MS. The $\alpha$ ion $(\mathrm{m} / \mathrm{z}$ 196) corresponds to an allylic cleavage to the erstwhile double bond and $\omega$ ion $(\mathrm{m} / \mathrm{z} 220)$ follows unique fragmentation pattern of $\Delta 5$ monoenes at $\mathrm{C}_{2-3}$.....

Supplementary Figure 2. Rules of $\mathrm{H}$ transfer upon collision activation of methylene interrupted fatty acid methyl esters (FAME) $[M+54]^{+}$ion. Double bond associated $\mathrm{CH}_{2} \mathrm{CN}^{+} \mathrm{CH}_{2}(\mathrm{~m} / \mathrm{z}=54)$ adduct is not shown. a) Monoenes, 18:1(9Z) shown. b) Dienes 18:2(9Z,12Z). c) Conjugated dienes 18:2(9Z,11Z).

d) Polyenes 18:3(9Z,12Z,15Z).

Supplementary Figure 3. Rules of $\mathrm{H} \bullet$ flow upon collision activation of $\Delta 5$ and $\Delta 7 \mathrm{PMI}$ fatty acid methyl esters (FAME). Double bond associated $\mathrm{CH}_{2} \mathrm{CN}^{+} \mathrm{CH}_{2}(\mathrm{~m} / \mathrm{z}=54)$ adduct is not shown. a) 18:2(5Z,11Z). b) 20:2(5Z,11Z). c) 22:2(5Z,13Z). d) 20:2(5Z,14Z). e) 20:3(5Z,11Z,14Z). f) $20: 3(7 Z, 11 Z, 14 Z)$.

Supplementary Figure 4. TIC chromatograms of a) pine nut, b) ginkgo nut and c) Thuja occidentalis. 
39 with $\Delta 5$ isolated double bond ( $\omega$ diagnostic ions generalized).

\begin{tabular}{|c|c|c|c|c|c|c|}
\hline \multirow{2}{*}{ FAME } & \multicolumn{2}{|c|}{$\alpha$ Diagnostic lons } & \multirow[b]{2}{*}{$-\left(\mathrm{CH}_{2}\right)_{4^{-}}$} & \multirow[b]{2}{*}{$-\left(\mathrm{CH}_{2}\right)_{5-}^{-}$} & \multirow[b]{2}{*}{$-\left(\mathrm{CH}_{2}\right)_{6^{-}}$} & \multirow[b]{2}{*}{$-(\mathrm{CH} 2)_{7^{-}}$} \\
\hline & $-\left(\mathrm{CH}_{2}\right)_{2^{-}}$ & $-\left(\mathrm{CH}_{2}\right)_{3-}$ & & & & \\
\hline$D B$ positions, dienes & 5,9 & 5,10 & 5,11 & 5,12 & 5,13 & 5,14 \\
\hline \multirow[t]{2}{*}{ Dienes } & 236 & 250 & 278 & 292 & 306 & 196 \\
\hline & 250 & 264 & & & & 320 \\
\hline$D B$ positions, trienes & $5,9,12$ & $5,10,13$ & $5,11,14$ & $5,12,15$ & $5,13,16$ & $5,14,17$ \\
\hline \multirow[t]{2}{*}{ Trienes } & 234 & 248 & 262 & $(276)$ & $(290)$ & $(304)$ \\
\hline & 276 & 290 & 304 & 318 & 332 & 346 \\
\hline$D B$ positions, tetraenes & $5,9,12,15$ & $5,10,13,16$ & $5,11,14,17$ & $5,12,15,18$ & $5,13,16,19$ & $5,14,17,20$ \\
\hline \multirow[t]{3}{*}{ Tetraenes } & 274 & 288 & 302 & 316 & 330 & 344 \\
\hline & \multicolumn{2}{|c|}{$\omega$ Diagnostic lons } & & & & \\
\hline & $-\left(\mathrm{CH}_{2}\right)_{2^{-}}$ & $-\left(\mathrm{CH}_{2}\right)_{3}-$ & $-\left(\mathrm{CH}_{2}\right)_{4^{-}}$ & $-\left(\mathrm{CH}_{2}\right)_{5^{-}}$ & $-\left(\mathrm{CH}_{2}\right)_{6^{-}}$ & $-(\mathrm{CH} 2)_{7-}$ \\
\hline$D B$ positions, dienes & 5,9 & 5,10 & 5,11 & 5,12 & 5,13 & 5,14 \\
\hline \multirow[t]{5}{*}{ Dienes } & M-74 & $M-74$ & M-74 & $M-74$ & M-74 & M-74 \\
\hline & $M-128$ & $M-128$ & $M-128$ & $M-128$ & M-128 & $M-128$ \\
\hline & $M-140$ & $(\mathrm{M}-142)$ & (M-142) & $(\mathrm{M}-182)$ & M-196 & $M-210$ \\
\hline & $M-142$ & & & & & \\
\hline & $M-154$ & & & & & \\
\hline$D B$ positions, trienes & $5,9,12$ & $5,10,13$ & $5,11,14$ & $5,12,15$ & $5,13,16$ & $5,14,17$ \\
\hline \multirow[t]{4}{*}{ Trienes } & M-74 & $M-74$ & M-74 & $M-74$ & $M-74$ & M-74 \\
\hline & $M-128$ & $M-128$ & $M-128$ & $M-128$ & $M-128$ & $M-128$ \\
\hline & $M-142$ & $M-142$ & $(M-142)$ & M-196 & $M-210$ & $M-224$ \\
\hline & $M-154$ & $M-168$ & $M-182$ & & & \\
\hline$D B$ positions, tetraenes & $5,9,12,15$ & $5,10,13,16$ & $5,11,14,17$ & $5,12,15,18$ & $5,13,16,19$ & $5,14,17,20$ \\
\hline \multirow[t]{4}{*}{ Tetraenes } & $M-74$ & M-74 & M-74 & $M-74$ & $M-74$ & M-74 \\
\hline & $M-128$ & $M-128$ & $M-128$ & $M-128$ & $M-128$ & $M-128$ \\
\hline & M-142 & $M-142$ & $(M-142)$ & M-196 & $M-210$ & $M-224$ \\
\hline & $M-154$ & $M-168$ & M-182 & & & \\
\hline
\end{tabular}

40 Note:

41 Transition: [M+54]-->products

42 Numbers in parenthesis may be not present at prominent intensity

43 Numbers of bold font is actual observations 
45 Supplementary Table 2. List of expected SMCI-MS/MS diagnostic ions for PMI-FAME with $\Delta 4$ isolated

double bond.

\begin{tabular}{|c|c|c|c|c|c|c|}
\hline \multirow{2}{*}{ FAME } & \multicolumn{2}{|c|}{$\alpha$ Diagnostic lons } & \multirow[b]{2}{*}{$-\left(\mathrm{CH}_{2}\right)_{4^{-}}$} & \multirow[b]{2}{*}{$-\left(\mathrm{CH}_{2}\right)_{5^{-}}$} & \multirow[b]{2}{*}{$-\left(\mathrm{CH}_{2}\right)_{6^{-}}$} & \multirow[b]{2}{*}{$-(\mathrm{CH} 2)_{7^{-}}$} \\
\hline & $-\left(\mathrm{CH}_{2}\right)_{2}^{-}$ & $-\left(\mathrm{CH}_{2}\right)_{3^{-}}$ & & & & \\
\hline DB positions, dienes & 4,8 & 4,9 & 4,10 & 4,11 & 4,12 & 4,13 \\
\hline \multirow[t]{2}{*}{ Dienes } & 222 & 236 & 264 & 278 & 292 & 182 \\
\hline & 236 & 250 & & & & 306 \\
\hline DB positions, trienes & $4,8,11$ & $4,9,12$ & $4,10,13$ & $4,11,14$ & $4,12,15$ & $4,13,16$ \\
\hline \multirow[t]{2}{*}{ Trienes } & 220 & 234 & 248 & 262 & 276 & 290 \\
\hline & 262 & 276 & 290 & 304 & 318 & 332 \\
\hline DB positions, tetraenes & $4,8,11,14$ & $4,9,12,15$ & $4,10,13,16$ & $4,11,14,17$ & $4,12,15,18$ & $4,13,16,19$ \\
\hline \multirow[t]{3}{*}{ Tetraenes } & 260 & 274 & 288 & 302 & 316 & 330 \\
\hline & \multicolumn{2}{|c|}{$\omega$ Diagnostic lons } & & & & \\
\hline & $-\left(\mathrm{CH}_{2}\right)_{2^{-}}$ & $-\left(\mathrm{CH}_{2}\right)_{3}^{-}$ & $-\left(\mathrm{CH}_{2}\right)_{4}^{-}$ & $-\left(\mathrm{CH}_{2}\right)_{5^{-}}$ & $-\left(\mathrm{CH}_{2}\right)_{6}{ }^{-}$ & $-(\mathrm{CH} 2)_{7^{-}}$ \\
\hline DB positions, dienes & 4,8 & 4,9 & 4,10 & 4,11 & 4,12 & 4,13 \\
\hline \multirow[t]{5}{*}{ Dienes } & $M-74$ & $M-74$ & $\mathrm{M}-74$ & $M-74$ & $M-74$ & $M-74$ \\
\hline & $M-114$ & $\mathrm{M}-114$ & $M-114$ & $M-114$ & $M-114$ & $\mathrm{M}-114$ \\
\hline & $M-126$ & (M-128) & (M-128) & (M-168) & M-182 & M-196 \\
\hline & $M-128$ & & & & & \\
\hline & $M-140$ & & & & & \\
\hline$D B$ positions, trienes & $4,8,11$ & $4,9,12$ & $4,10,13$ & $4,11,14$ & $4,12,15$ & $4,13,16$ \\
\hline \multirow[t]{4}{*}{ Trienes } & $M-74$ & $M-74$ & $M-74$ & $M-74$ & $M-74$ & $M-74$ \\
\hline & M-114 & $\mathrm{M}-114$ & M-114 & $\mathrm{M}-114$ & M-114 & $\mathrm{M}-114$ \\
\hline & $M-128$ & $M-128$ & (M-128) & M-182 & M-196 & $M-210$ \\
\hline & $M-140$ & $M-154$ & $M-168$ & & & \\
\hline$D B$ positions, tetraenes & $4,8,11,14$ & $4,9,12,15$ & $4,10,13,16$ & $4,11,14,17$ & $4,12,15,18$ & $4,13,16,19$ \\
\hline \multirow[t]{4}{*}{ Tetraenes } & $\mathrm{M}-74$ & $\mathrm{M}-74$ & & $\mathrm{M}-74$ & $M-74$ & $M-74$ \\
\hline & M-114 & $\mathrm{M}-114$ & $\mathrm{M}-114$ & $\mathrm{M}-114$ & M-114 & $\mathrm{M}-114$ \\
\hline & $M-128$ & $M-128$ & (M-128) & M-182 & M-196 & $M-210$ \\
\hline & M-140 & M-154 & M-168 & & & \\
\hline
\end{tabular}

47

Note:

48 Transition: [M+54]-->products

Numbers in parenthesis may be not present at prominent intensity 
51 double bond.

\begin{tabular}{|c|c|c|c|c|c|c|}
\hline \multirow{2}{*}{ FAME } & \multicolumn{2}{|c|}{$\alpha$ Diagnostic lons } & \multirow[b]{2}{*}{$-\left(\mathrm{CH}_{2}\right)_{4^{-}}$} & \multirow[b]{2}{*}{$-\left(\mathrm{CH}_{2}\right)_{5^{-}}$} & \multirow[b]{2}{*}{$-\left(\mathrm{CH}_{2}\right)_{6^{-}}$} & \multirow[b]{2}{*}{$-(\mathrm{CH} 2)_{7^{-}}$} \\
\hline & $-\left(\mathrm{CH}_{2}\right)_{2^{-}}$ & $-\left(\mathrm{CH}_{2}\right)_{3^{-}}$ & & & & \\
\hline DB positions, dienes & 6,10 & 6,11 & 6,12 & 6,13 & 6,14 & 6,15 \\
\hline \multirow[t]{2}{*}{ Diene } & 250 & 264 & 292 & 306 & 320 & 210 \\
\hline & 264 & 278 & & & & 334 \\
\hline DB positions, trienes & $6,10,13$ & $6,11,14$ & $6,12,15$ & $6,13,16$ & $6,14,17$ & $6,15,18$ \\
\hline \multirow[t]{2}{*}{ Triene } & 248 & 262 & 276 & 290 & 304 & 318 \\
\hline & 290 & 304 & 318 & 332 & 346 & 360 \\
\hline DB positions, tetraenes & $6,10,13,16$ & $6,11,14,17$ & $6,12,15,18$ & $6,13,16.19$ & $6,14,17,20$ & $6,15,18,21$ \\
\hline \multirow[t]{3}{*}{ Tetraene } & 288 & 302 & 316 & 330 & 344 & 358 \\
\hline & \multicolumn{2}{|c|}{$\omega$ Diagnostic lons } & & & & \\
\hline & $-\left(\mathrm{CH}_{2}\right)_{2^{-}}$ & $-\left(\mathrm{CH}_{2}\right)_{3^{-}}$ & $-\left(\mathrm{CH}_{2}\right)_{4^{-}}$ & $-\left(\mathrm{CH}_{2}\right)_{5^{-}}$ & $-\left(\mathrm{CH}_{2}\right)_{6^{-}}$ & $-(\mathrm{CH} 2)_{7^{-}}$ \\
\hline DB positions, dienes & 6,10 & 6,11 & 6,12 & 6,13 & 6,14 & 6,15 \\
\hline \multirow[t]{4}{*}{ Diene } & M-142 & M-142 & M-142 & M-142 & M-142 & M-142 \\
\hline & $M-154$ & (M-156) & (M-156) & (M-196) & $M-210$ & M-224 \\
\hline & M-156 & & & & & \\
\hline & M-168 & & & & & \\
\hline DB positions, trienes & $6,10,13$ & $6,11,14$ & $6,12,15$ & $6,13,16$ & $6,14,17$ & $6,15,18$ \\
\hline \multirow[t]{3}{*}{ Triene } & M-142 & M-142 & $M-142$ & M-142 & M-142 & M-142 \\
\hline & (M-156) & (M-156) & (M-156) & $M-210$ & M-224 & M-238 \\
\hline & M-168(or M-170) & M-182 & M-196 & & & \\
\hline DB positions, tetraenes & $6,10,13,16$ & $6,11,14,17$ & $6,12,15,18$ & $6,13,16.19$ & $6,14,17,20$ & $6,15,18,21$ \\
\hline \multirow[t]{3}{*}{ Tetraene } & M-142 & M-142 & M-142 & M-142 & M-142 & $\mathrm{M}-142$ \\
\hline & (M-156) & (M-156) & (M-156) & M-210 & M-224 & M-238 \\
\hline & M-168(or M-170) & M-182 & M-196 & & & \\
\hline
\end{tabular}

52 Note:

53 Transition: [M+54]-->products

54 Numbers in parenthesis may be not present at prominent intensity 
55 Supplementary Table 4. List of observed and expected SMCI-MS/MS diagnostic ions for PMI-FAME

56 with $\Delta 7$ isolated double bond.

\begin{tabular}{|c|c|c|c|c|c|c|}
\hline \multirow{2}{*}{ FAME } & \multicolumn{2}{|c|}{$\alpha$ Diagnostic lons } & \multirow[b]{2}{*}{$-\left(\mathrm{CH}_{2}\right)_{4}-$} & \multirow[b]{2}{*}{$-\left(\mathrm{CH}_{2}\right)_{5^{-}}$} & \multirow[b]{2}{*}{$-\left(\mathrm{CH}_{2}\right)_{6^{-}}$} & \multirow[b]{2}{*}{$-(\mathrm{CH} 2)_{7}-$} \\
\hline & $-\left(\mathrm{CH}_{2}\right)_{2^{-}}$ & $-\left(\mathrm{CH}_{2}\right)_{3^{-}}$ & & & & \\
\hline $\begin{array}{l}\text { DB positions, } \\
\text { dienes }\end{array}$ & 7,11 & 7,12 & 7,13 & 7,14 & 7,15 & 7,16 \\
\hline \multirow[t]{2}{*}{ Diene } & 264 & 278 & 306 & 320 & 334 & 224 \\
\hline & 278 & 292 & & & & 348 \\
\hline $\begin{array}{l}\text { DB positions, } \\
\text { trienes }\end{array}$ & $7,11,14$ & $7,12,15$ & $7,13,16$ & $7,14,17$ & $7,15,18$ & $7,16,19$ \\
\hline \multirow[t]{2}{*}{ Triene } & 262 & 276 & 290 & 304 & 318 & 332 \\
\hline & 304 & 318 & 332 & 346 & 360 & 374 \\
\hline $\begin{array}{l}\text { DB positions, } \\
\text { tetraenes }\end{array}$ & $7,11,14,17$ & $7,12,15,18$ & $7,13,16,19$ & $7,14,17,20$ & $7,15,18,21$ & $7,16,19,22$ \\
\hline \multirow[t]{3}{*}{ Tetraene } & 302 & 316 & 330 & 344 & 358 & 372 \\
\hline & \multicolumn{2}{|c|}{$\omega$ Diagnostic lons } & & & & \\
\hline & $-\left(\mathrm{CH}_{2}\right)_{2-}^{-}$ & $-\left(\mathrm{CH}_{2}\right)_{3}-$ & $-\left(\mathrm{CH}_{2}\right)_{4}^{-}$ & $-\left(\mathrm{CH}_{2}\right)_{5-}^{-}$ & $-\left(\mathrm{CH}_{2}\right)_{6}{ }^{-}$ & $-(\mathrm{CH} 2)_{7-}^{-}$ \\
\hline $\begin{array}{l}\text { DB positions, } \\
\text { dienes }\end{array}$ & 7,11 & 7,12 & 7,13 & 7,14 & 7,15 & 7,16 \\
\hline \multirow[t]{4}{*}{ Diene } & M-156 & M-156 & M-156 & M-156 & M-156 & M-156 \\
\hline & M-168 & (M-170) & (M-170) & (M-210) & M-224 & $M-238$ \\
\hline & M-170 & & & & & \\
\hline & M-182 & & & & & \\
\hline $\begin{array}{l}\text { DB positions, } \\
\text { trienes }\end{array}$ & $7,11,14$ & $7,12,15$ & $7,13,16$ & $7,14,17$ & $7,15,18$ & $7,16,19$ \\
\hline \multirow[t]{3}{*}{ Triene } & M-156 & M-156 & M-156 & M-156 & M-156 & M-156 \\
\hline & (M-170) & $(\mathrm{M}-170)$ & (M-170) & M-224 & M-238 & M-252 \\
\hline & M-184 & M-196 & $\mathrm{M}-210$ & & & \\
\hline $\begin{array}{l}\text { DB positions, } \\
\text { tetraenes }\end{array}$ & $7,11,14,17$ & $7,12,15,18$ & $7,13,16,19$ & $7,14,17,20$ & $7,15,18,21$ & $7,16,19,22$ \\
\hline \multirow[t]{3}{*}{ Tetraene } & $M-156$ & M-156 & M-156 & M-156 & M-156 & M-156 \\
\hline & $(\mathrm{M}-170)$ & $(\mathrm{M}-170)$ & (M-170) & M-224 & M-238 & M-252 \\
\hline & M-184 & M-196 & $\mathrm{M}-210$ & & & \\
\hline
\end{tabular}

57

Note:

58 Transition: $[\mathrm{M}+54]-->$ products

59 Numbers in parenthesis may be not present at prominent intensity

Numbers of bold font is actual observations 
61 Supplementary Figure 1. Identification of 14:1( $\Delta 5)$ in a ruminant milk sample by CACI-MS/MS. The $\alpha$

62 ion $(\mathrm{m} / \mathrm{z}$ 196) corresponds to an allylic cleavage to the erstwhile double bond and $\omega$ ion $(\mathrm{m} / \mathrm{z} 220)$

63 follows unique fragmentation pattern of $\Delta 5$ monoenes at $\mathrm{C}_{2-3}$.

64

65

66

67

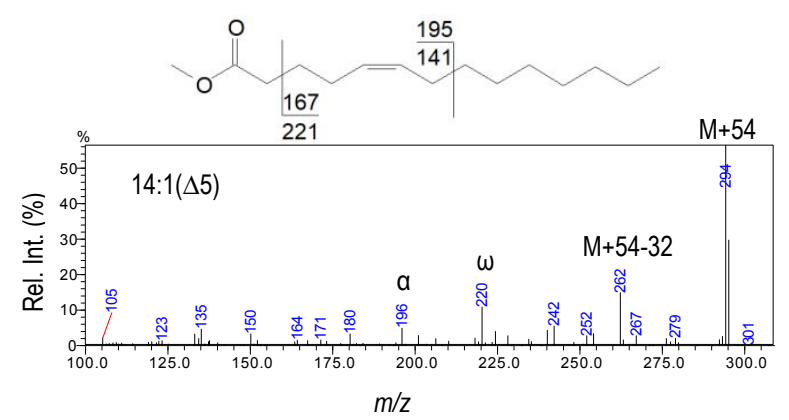


74 75 76 77 78 79 80 81 82 83

84

85 86

Supplementary Figure 2. Rules of $\mathrm{H}$ transfer upon collision activation of methylene interrupted fatty acid methyl esters (FAME) $[\mathrm{M}+54]^{+}$ion. Double bond associated $\mathrm{CH}_{2} \mathrm{CN}^{+} \mathrm{CH}_{2}(\mathrm{~m} / \mathrm{z}=54)$ adduct is not shown. a) Monoenes, 18:1(9Z) shown. b) Dienes 18:2(9Z,12Z). c) Conjugated dienes 18:2(9Z,11Z). d)

Polyenes 18:3(9Z,12Z,15Z).

a.

$\alpha$ fragments

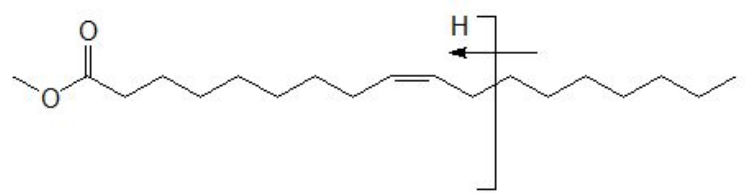

b.

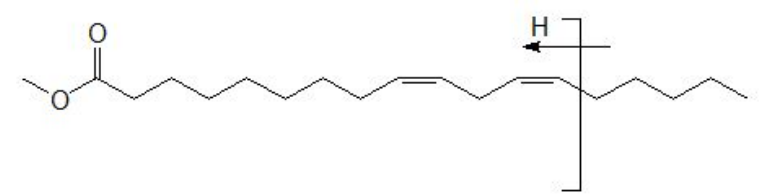

C.

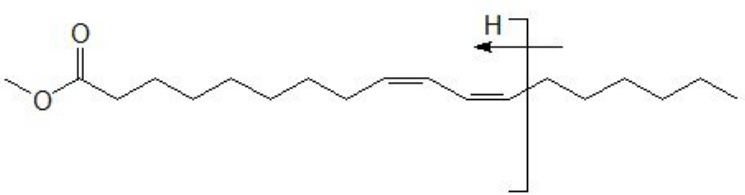

d.

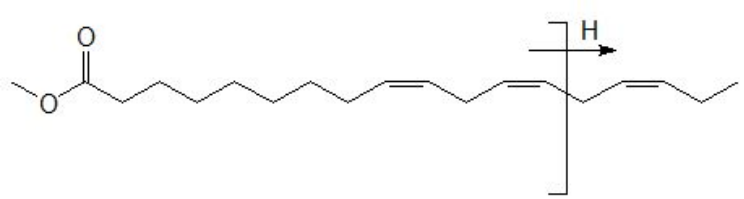

\section{$\omega$ fragments}
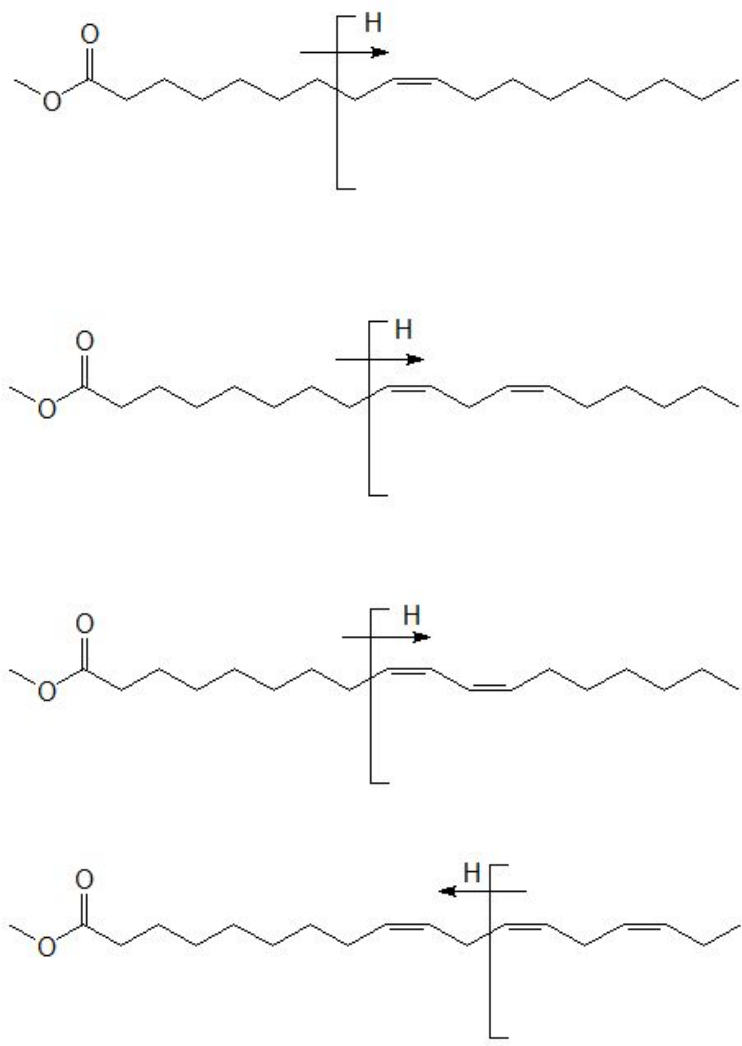
92

93

94

95

96

97

98

99

100

101

102

103

104

105

106

107

108

109

110

111

112

113

Supplementary Figure 3. Rules of $\mathrm{H} \bullet$ flow upon collision activation of $\Delta 5$ and $\Delta 7 \mathrm{PMI}$ fatty acid methyl esters (FAME). Double bond associated $\mathrm{CH}_{2} \mathrm{CN}^{+} \mathrm{CH}_{2}(\mathrm{~m} / \mathrm{z}=54)$ adduct is not shown. a) 18:2(5Z,11Z).

b) $20: 2(5 Z, 11 Z)$. c) $22: 2(5 Z, 13 Z)$. d) $20: 2(5 Z, 14 Z)$. e) $20: 3(5 Z, 11 Z, 14 Z)$. f) $20: 3(7 Z, 11 Z, 14 Z)$.

a fragments

$\omega$ fragments

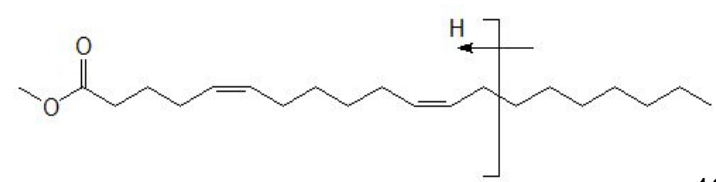

$18: 2(5 Z, 11 Z)$

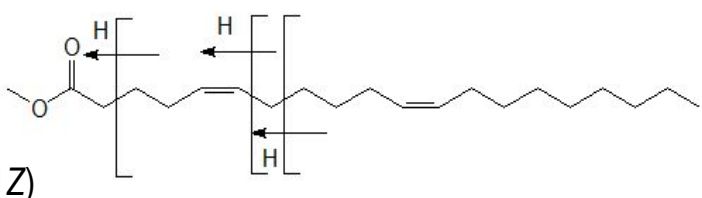

b.
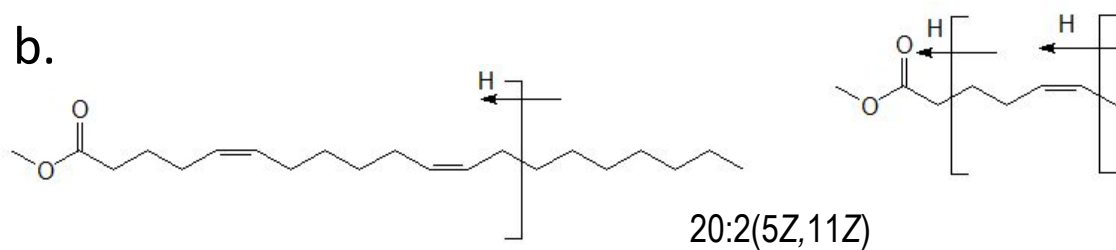

$20: 2(5 Z, 11 Z)$

C.

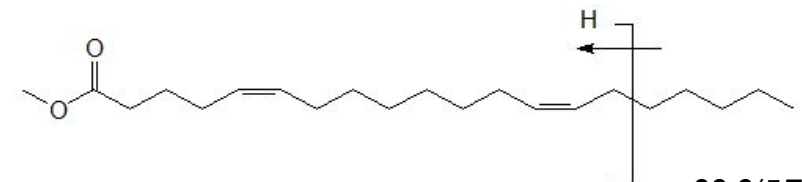

$22: 2(5 Z, 13 Z)$

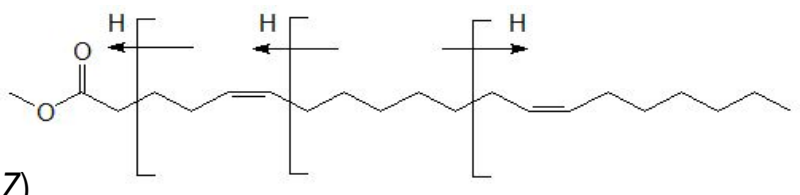

d.

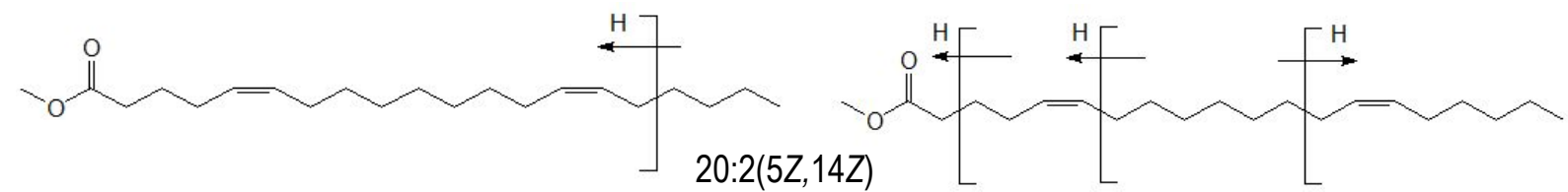

e.
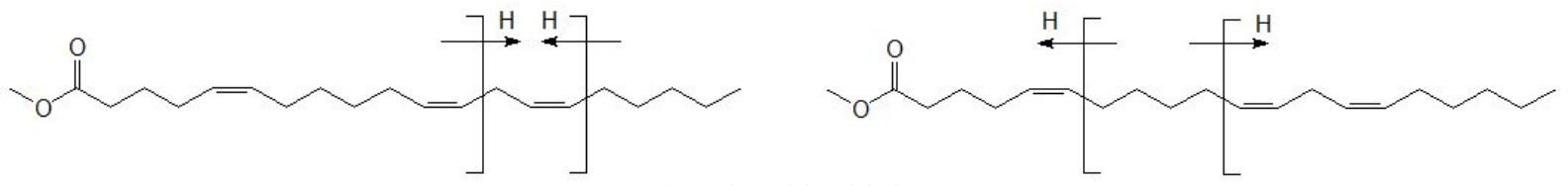

$20: 3(5 Z, 11 Z, 14 Z)$

f.

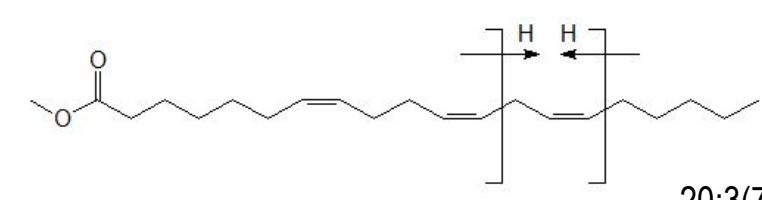

$20: 3(7 Z, 11 Z, 14 Z)$

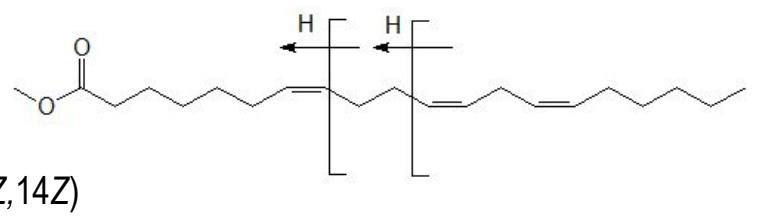




115
116
117
118
119
120
121
122

123 b.

132 C

133

134

135

\title{
136
}

.

133
134
135
136
137
138

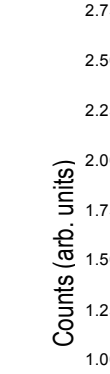

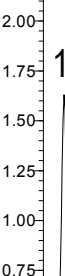

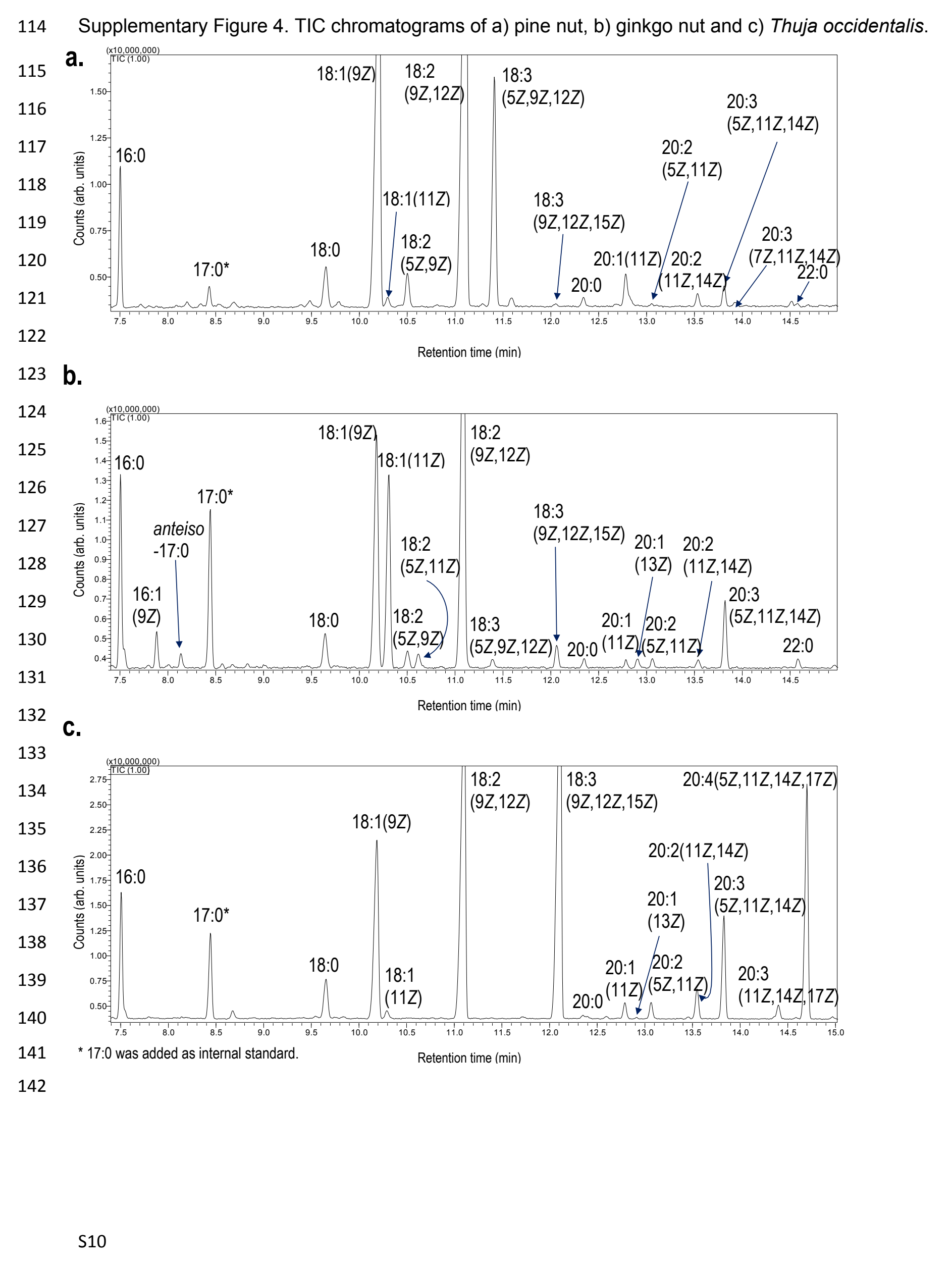

114 Supplementary Figure 4. TIC chromatograms of a) pine nut, b) ginkgo nut and c) Thuja occidentalis.
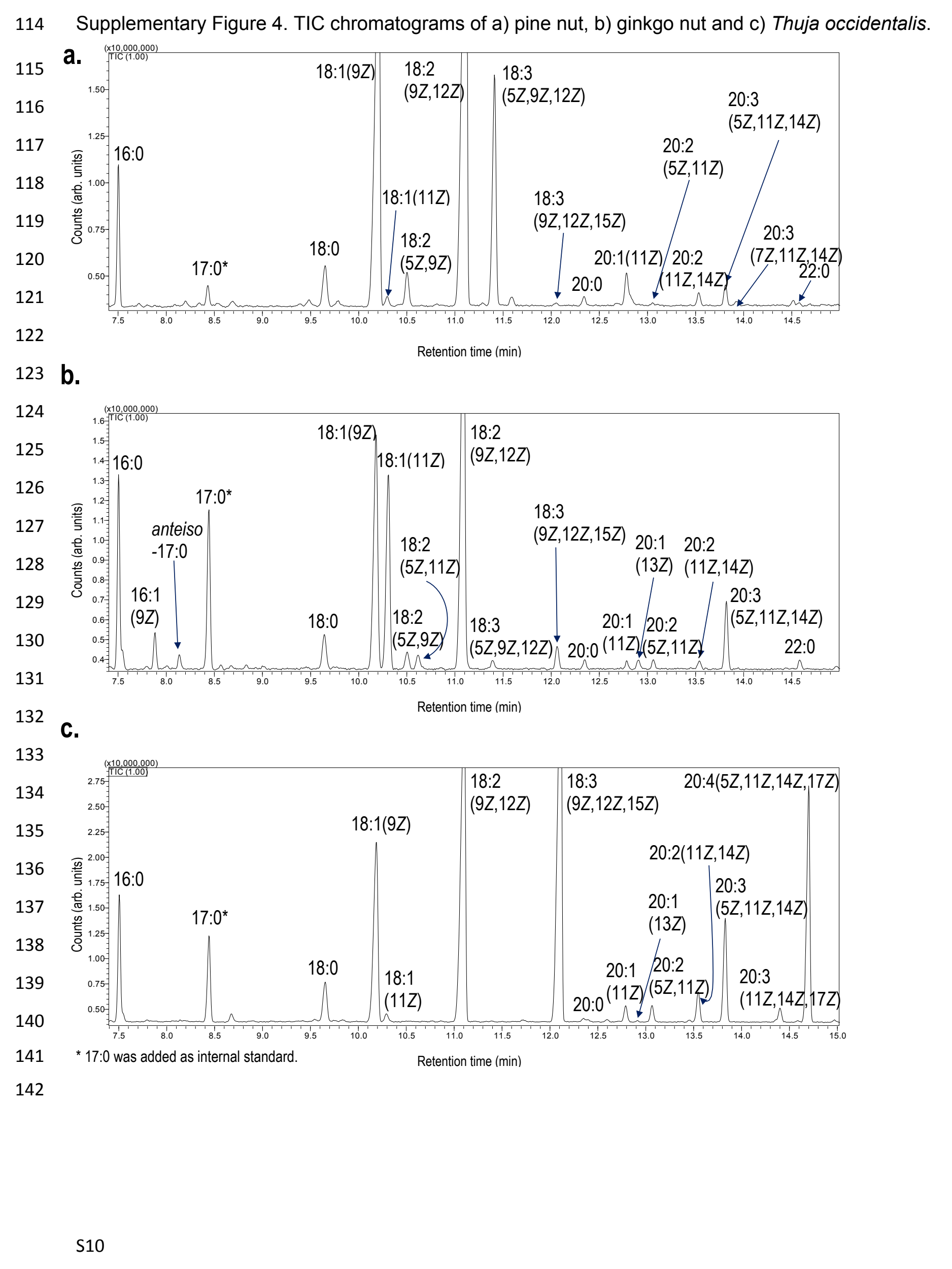$$
\text { 16:0 }
$$
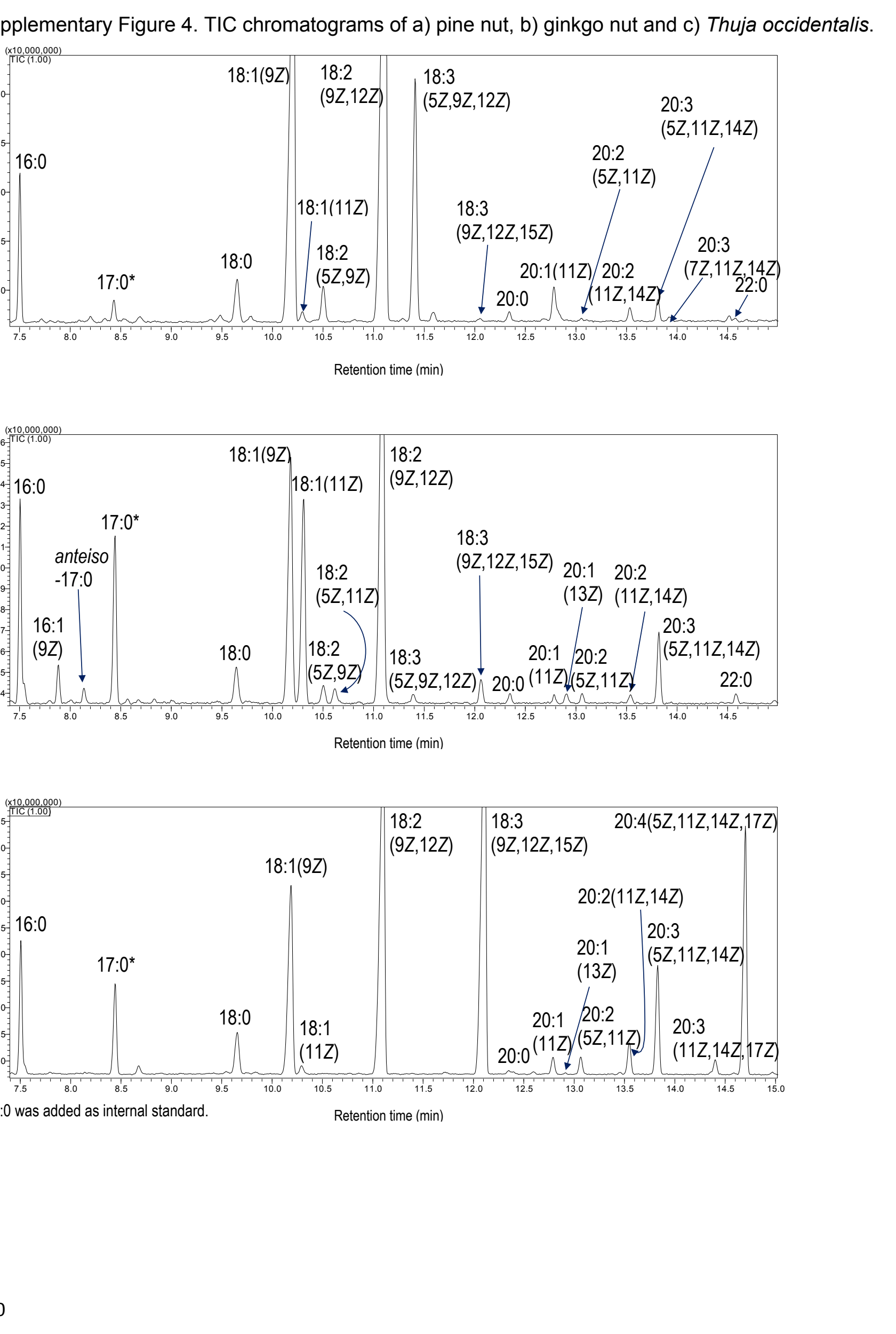

Retention time (min)

142

(n)

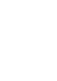

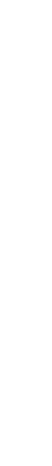

\begin{abstract}
* 17:0 was added as internal standard.
\end{abstract}

de

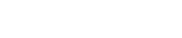

139

2

C. 